\title{
Risk factors associated with infantile spasms: a hospital-based case-control study in Taiwan
}

\author{
Horng-Huei Liou ${ }^{a}$, Pei-Ching Oon c,f Haung-Chi Lin ${ }^{\mathrm{d}}$, Pen-Jung Wang b,e, \\ Tony Hsiu-Hsi Chen ${ }^{\mathrm{f}, *}$ \\ a Department of Neurology and Pharmacology, \\ National Taiwan University Hospital and National Taiwan University College of Medicine, 7 Chung San South Road, \\ Taipei, Taiwan, ROC \\ ${ }^{\mathrm{b}}$ Department of Pediatrics, National Taiwan University Hospital and National Taiwan University College of Medicine, Taiwan, \\ ROC \\ ${ }^{\mathrm{c}}$ Department of Internal Medicine, National Taiwan University Hospital, 7 Chung San South Road, Taipei, Taiwan, ROC \\ d Department of Pediatrics, En Chu Kong Hospital, 399 Fuhfing Road, Sanhsia, Taipei 237, Taiwan, ROC \\ e Department of Pediatrics, College of Medicine, Tzu Chi University, 701, Section 3, Chung-Yan Road, Hualien, Taiwan, ROC \\ ${ }^{\mathrm{f}}$ Graduate Institute of Preventative Medicine, College of Public Health, National Taiwan University, Room 207, No. 19, \\ Hsu-Chow Road, Taipei 100, Taiwan, ROC
}

Received 16 March 2001; received in revised form 9 July 2001; accepted 20 July 2001

\begin{abstract}
We investigated the risk factors associated with infantile spasms (IS) by a hospital-based case-control study in Taiwan. Twenty-five patients with IS were recruited from one medical center (National Taiwan University Hospital) between 1990 and 1997. Based on a close-structured questionnaire, standardized interviews were carried out to obtain information on risk factors associated with IS. Two comparison groups are used, including a total of 106 subjects in the Disease Control group, and 139 subjects in the Normal Control group. Unconditional logistic regression is used to calculate odds ratios (OR) and 95\% confidence interval (CI). Univariate analysis revealed gestational age, congenital cerebral anomalies, tuberous sclerosis (TS), asphyxia, febrile seizure, and developmental delay (before onset of spasm) were at increased risk of IS. After adjustment of multiple risk factors through unconditional logistic regression, significant risk factors for IS include congenital cerebral anomalies, TS, asphyxia, postterm, and developmental delay were highly associated with IS. The risk factors of IS may closely relate to underlying neurological abnormalities. Our results are consistent with the previous findings. (C) 2001 Elsevier Science B.V. All rights reserved.
\end{abstract}

Keywords: Infantile spasms; Risk factors; Case-control study; Taiwan

* Corresponding author. Tel.: + 886-2-2358-7620; fax: + 886-2-2358-7707.

E-mail address: stony@episerv.cph.ntu.edu.tw (T.H.-H. Chen). 


\section{Introduction}

Infantile spasms (IS) is a severe age-specific epileptic syndrome which is intractable to standard anticonvulsant drug therapy and carries poor prognosis for normal intellectual development (Hrachovy and Frost, 1989; Hauser, 1994; Dulac et al., 1997). Annual incidence rate of IS has been reported to range from 0.16 to 0.42 per thousand live births (Howitz and Platz, 1978; Riikonen and Donner 1979; Luthvigsson et al., 1994; Trevathan et al., 1999). Prevalence rate of IS has also been reported to range from 0.14 to 0.21 per thousand children aged from birth through 9 years (Cowan et al., 1989; Trevathan et al., 1999; Debrock et al., 2000). The reported factors which predispose to IS include birth trauma, hypoxia-ischemic encephalopathy, central nervous system (CNS) infection, brain malformations and genetic or metabolic disease such as tuberous sclerosis (TS), and phenylketonuria (Ohtahara, 1984; Filipek et al., 1987; Cusmai et al., 1993; Dulac et al., 1993; Bobo et al., 1994; Hou et al., 1994; Chiron et al., 1996; Rantala et al., 1996; Dulac et al., 1997; Ozawa et al., 1998; Watanabe, 1998). It should be noted that most studies on IS have been focused on the Western countries, and very few have addressed among the Orientals. Thus, it is timely to identify risk factors associated with IS in Taiwan.

Previous reported factors responsible for IS were postulated on the basis of case series rather than well-designed epidemiological studies. Consequently, the empirical evidence on risk factors associated with IS was rather meager and limited. Even though some epidemiological studies had been conducted to elucidate the association of relevant risk factors with IS, the credibility of their results was often questioned due to the lack of control groups. Knowing that the risk factors accounting for IS in Western countries may differ from those in Oriental countries, we deemed it worthwhile to conduct a case-control study using multiple comparison groups among people in Taiwan. The aim of our study was therefore to identify risk factors using a hospital-based casecontrol study.

\section{Materials and methods}

The diagnosis of IS was made by the presence of clinical history of spasm, and characteristic electroencephalogram (EEG) with or without psychomotor retardation after onset of spasms. The spasms were classified as flexor, extensor or mixed according to Kellaway et al. (1979). The EEG was classified as either hypsarrhythmic or modified hypsarrhythmic by the criteria of Gibbs and Gibbs (1952) and Hrachovy et al. (1984), respectively. Besides, subjects having spasms and psychomotor retardation with multifocal EEG activity were included (Fois et al., 1984). A total of 25 IS cases have been recruited with above criteria by pediatric neurologists from 1990 through 1997 in National Taiwan University Hospital. There are five cases fulfilling all the diagnostic criteria of triad, 19 cases meeting both clinical spasms and EEG hypsarrhythmia or modified hypsarrhythmia, and one patient having spasms, psychomotor retardation and multifocal EEG activity.

A hospital-based case-control study using approximately 1:4 ratio of case: control was conducted from August 1999 to July 2000. Two types of control were chosen as comparison groups: the 'Normal Control' group, derived from all neonates in the contemporary period as the IS group at the same medical center; and the 'Disease Control' group. The rationale for using the Disease Control group is that certain risk factors such as cerebral palsy (CP) and TS are rare and can be hardly identified if the only Normal Control group is adopted. In order to be efficient when investigating the role of these factors in the development of IS, a set of random samples from the Disease Control was therefore considered. It should be noted that the Disease Control group not only consists of the diseases that represents risk factors associated with IS but also includes those unrelated to IS. The Disease Control group consists of prematurity, small for gestational age, asphyxia, Down's syndrome, chromosome disorder, CP, CNS infection, traumatic brain injury (subarachnoid hemorrhage and subdural hemorrhage), TS and other neurocutaneous diseases, white matter diseases, progressive encephalopathy 
of infancy, periventrivular leukomalacia, congenital heart diseases and other congenital cerebral anomalies including neuronal migration disorder, holoprosencephaly, lissencephaly, and brain atrophy. Frequency matching on year of birth for Control groups was performed to prevent the imbalance between cases and the Control groups with respect to age distribution.

Mothers of every subject in IS group and control groups were contacted by trained physicians on the telephone to inquire them whether they were willing to participate in this study. All attendants were interviewed with a closed-structured questionnaire. Information of the questionnaire consists of demographic characteristics, family history of epilepsy or seizures, congenital cerebral anomaly and psychiatric diseases, prenatal complications such as eclampsia, gestational history, and infants' disease history, including asphyxia, chromosome disorder, TS, neurofibromatosis, CNS infection, head injury, febrile seizure before onset of spasms, congenital cerebral anomalies, and developmental delay before onset of spasms. In addition, long-term neurodevelopmental outcomes of these cases were also collected.

The content validity of questionnaire has been checked on the basis of expert opinions, including one expert specialized in epidemiology and three in neurology. A total of 106 subjects and a total of 139 subjects in Disease Control group and in Normal Control group were willing to answer the questionnaire during the interview. The telephone interviews were standardized before interviewing and conducted by trained physicians.

In addition to questionnaire interview, clinical history, developmental and laboratory testing including computed tomography or magnetic resonance imaging of the head, metabolic studies (serum amino acids, urine organic acids), and chromosomal studies were collected by chart review to establish the diagnosis of associated diseases with IS. Cases were designated symptomatic if there was a known etiology or signs of previous brain damage and cryptogenic if these factors were lacking (Commission on Classification and Terminology of the International League Against Epilepsy, 1989). Asphyxia was defined according to overt prenatal, perinatal or postnatal clinical event. Perinatal asphyxia was defined as an Apgars score of 5 or less at one and five minutes (Rantala et al., 1996; Hanjal et al., 1999; Shevell et al., 1999). Febrile seizure was defined as seizure during febrile stage without evidence of intracranial infection or definable causes. In IS cases, febrile seizure should occur prior to onset of spasms. Young infant seizure was defined as seizure before the age of 3 months.

Univariate and multivariate analysis using unconditional logistic regression was performed to identify significant factors accounting for IS. Statistical significant level was set up at level of 0.05 .

\section{Results}

Among the 25 patients with IS, 21 (84\%) cases are etiologically symptomatic and four $(16 \%)$ cases are etiologically cryptogenic. The associated diseases of 21 symptomatic cases are: TS (6), asphyxia (4), prematurity (2), traumatic brain injury (1), hydrocephaly (1), early myoclonic encephalopathy (1), congenital infection (1), other congenital cerebral anomalies including $18 \mathrm{p}$-syndrome with holoprosencephaly (1), left temporal lobe dysgenesis (1), diffuse brain atrophy (1), and megalencephaly (1) and one case diagnosed as cerebral palsy without identified causes. Table 1 shows the distribution of age, sex and associated diseases in IS cases.

Table 2 shows the distribution of age onset for IS group and for the Disease Control group. Univariates analysis of significant risk factors for IS is also presented. Regarding age of onset, about $78 \%$ of the IS group have disease onset between 3 and 8 months. The corresponding figure for Disease Control group is $19 \%$. As expected, the majority of Normal Control group are born in term whereas about one in three of Disease Control group and $16 \%$ of IS were delivered before 36 weeks of conception (preterm).

As regards family history, IS is more likely to have family history of psychiatric disease than two other groups. The odds ratios (OR) for IS against Disease Control and Normal Control are $2.25(95 \% \mathrm{CI}=0.52-9.70)$ and $3.02(95 \% \mathrm{CI}=$ 
$0.70-12.98)$. No subject has family history of seizure or epilepsy in IS group.

Congenital cerebral anomaly is more frequent in the IS group and the Disease Control group than in the Normal Control group. Compared to the Normal Control, the OR for congential hydrocephaly in IS group is $5.75(95 \% \mathrm{CI}=0.22-$ 148.62). The corresponding OR for cerebral anomaly other than hydrocephaly and microcephaly is $4.60(95 \% \mathrm{CI}=0.96-21.97)$. The IS group is approximately four times $(\mathrm{OR}=3.73$, $95 \% \mathrm{CI}=1.26-11.10$ ) more likely to have TS than the Disease Control group.

IS and Disease Control group are more susceptible to asphyxia than the Normal Control group. The percentage is higher in the Disease Control group $(31.1 \%)$ than in the IS group (16.7\%). Compared with Normal Control, there is a 14-fold $(\mathrm{OR}=13.7,95 \% \mathrm{CI}=2.36-79.71)$ risk for as-

\section{Table 1}

Distribution of age and sex in infantile spasms (IS) and the diseases associate with symptomatic IS cases

\begin{tabular}{|c|c|c|}
\hline Infantile spasms & Case no. $(\%)$ & \\
\hline Age (years) & Male & Female \\
\hline $0-3$ & 2 & 3 \\
\hline $4-6$ & 6 & 7 \\
\hline $7-9$ & 4 & 3 \\
\hline Symptomatic & $21(84.0 \%)$ & \\
\hline Tuberous sclerosis & 6 & \\
\hline Asphyxia & 4 & \\
\hline Prematurity & 2 & \\
\hline $\begin{array}{l}\text { Traumatic brain injury } \\
\text { (subdural hemorrhage) }\end{array}$ & 1 & \\
\hline Hydrocephaly & 1 & \\
\hline $\begin{array}{l}\text { Early myoclonic } \\
\text { encephalopathy }\end{array}$ & 1 & \\
\hline Congenital infection & 1 & \\
\hline \multicolumn{3}{|l|}{ Congenital cerebral anomaly } \\
\hline $\begin{array}{l}\text { 18p-syndrome with } \\
\text { holoprosencephaly }\end{array}$ & 1 & \\
\hline $\begin{array}{l}\text { Left temporal lobe } \\
\text { dysgenesis }\end{array}$ & 1 & \\
\hline Diffuse brain atrophy & 1 & \\
\hline Megalencephaly & 1 & \\
\hline $\begin{array}{l}\text { Cerebral palsy with unknown } \\
\text { causes }\end{array}$ & 1 & \\
\hline Cryptogenic & $4(16.0 \%)$ & \\
\hline Total & 25 & \\
\hline
\end{tabular}

phyxia in the IS group. The risk is lower in the IS group than in the Disease Control group.

Febrile seizures tend to be more frequent in the IS group than two other control groups. The IS has 4-fold risk $(\mathrm{OR}=4.04,95 \% \mathrm{CI}=1.32-12.40)$ and 3 -fold risk $(\mathrm{OR}=3.03,95 \% \mathrm{CI}=0.98-9.34)$ for febrile seizure as compared with Normal Control and Disease Control, respectively. The IS group is much more likely to have developmental delay than the Disease Control group $(\mathrm{OR}=2.11$, $95 \% \mathrm{CI}=0.87-5.14)$ and the Normal Control group $(\mathrm{OR}=100.50,95 \% \mathrm{CI}=20.10-502.40)$.

The IS group has a higher proportion of young infant seizure than the two control groups. However, no statistically significance has been found. The occurrence of CNS infection and head injury in the IS group is not significantly higher than the other groups.

Table 3 shows results of multivariate analysis for the adjusted OR using IS versus Normal Control by incorporating significant factors in the univariate analysis plus age and sex. After adjustment for variables in each other, significant factors still persist including developmental delay (adjusted OR $=30.12,95 \% \mathrm{CI}=5.58-162.49$ ), asphyxia (adjusted $\mathrm{OR}=29.57,95 \% \quad \mathrm{CI}=3.04-$ 287.41), and congenital cerebral anomalies (adjusted $\mathrm{OR}=4.51,95 \% \mathrm{CI}=1.02-19.94$ ). Results of multivariate analysis for the adjusted OR using IS versus Disease Control group are listed in Table 4. Adjusted OR for significant factors are $15.61(95 \% \mathrm{CI}=1.37-178.26)$ for postterm and $9.05(95 \% \mathrm{CI}=2.24-36.59)$ for $\mathrm{TS}$.

We also collected information on prognosis of IS. Among 25 interviewed cases, 46\% still had intractable seizures, $77 \%$ had developmental delay or mental retardation and $60 \%$ have suffered from cerebral palsy (CP) or motor handicap.

\section{Discussion}

To the best of our knowledge, this is the first study to use a hospital-based case-control study to identify factors associated with IS in oriental countries. In the multiple conditional logistic regression analysis, we found children with TS, congenital cerebral anomalies, asphyxia, post- 
Table 2

Univariate analysis for the association between infantile spasms and risk factors

\begin{tabular}{lccc}
\hline Variable & $\begin{array}{l}\text { Infantile spasms (IS) } \\
N(\%)\end{array}$ & $\begin{array}{l}\text { Disease controls } \\
N(\%)\end{array}$ & $\begin{array}{l}\text { Norm } \\
N(\%)\end{array}$ \\
\hline Age of onset & $(m / o)$ & & \\
$0-3$ & 0 & $35(46.7)$ & 0 \\
$>3-8$ & $18(78.3)$ & $14(18.6)$ & 0 \\
$>8-12$ & $4(17.4)$ & $5(6.7)$ & 0 \\
$>12$ & $1(4.3)$ & $12(16.0)$ & 0 \\
Premature & 0 & $9(12.0)$ & 0 \\
NK & 2 & 31 & 0
\end{tabular}

Family history of psychiatric disease

Yes $\quad 3(12.0)$

No $22(88.0)$

$6(5.7)$

$99(94.3)$

3 (2.8)

$103(97.2)$

$35(34.0)$

$67(65.0)$

$1(1.0)$

$6(5.7)$

$100(94.3)$

$24(96.0)$

No

Microencephaly

Yes $0(0.0)$

No $25(100.0)$

Congenital cerebral anomaly ${ }^{\mathrm{a}}$

Yes 3 (12.0)

No $22(88.0)$

Tuberous sclerosis

Yes 7 (28.0)

No $18(72.0)$

Asphyxia

Yes 4 (16.7)

No $20(83.3)$

Febrile seizure

Yes 6 (24.0)

No $19(76.0)$

CNS infection

$\begin{array}{lrl}\text { Yes } & 1(4.0) \\ \text { No } & 24 & (96.0)\end{array}$

Head injury

Yes $1(4.0)$

No $24(96.0)$

Young infant seizure

Yes $\quad 4$ (16.7)

No $19(79.1)$

Developmental delay

Yes $15(60.0)$

Normal controls IS vs. disease control OR $(95 \% \mathrm{CI})$
IS vs. normal control OR $(95 \% \mathrm{CI})$

$\begin{array}{cc}2(1.9) & 0(0.0) \\ 104(98.1) & 139(100.0)\end{array}$

$21(19.8)$

$85(80.2)$

4 (2.9)

$135(97.1)$

$10(9.4)$

96 (90.6)

0

$139(100.0)$

$33(31.1)$

73 (68.9)

$2(1.4)$

137 (98.6)

$10(9.4)$

$96(90.6)$

$10(7.2)$

$128(92.8)$

3 (2.9)

$102(97.1)$

5 (3.6)

132 (96.4)

12 (11.3)

94 (88.7)

2 (1.5)

135 (98.5)

$11(10.4)$

95 (89.6)

0

139 (100.0)

44 (41.5)

62 (58.5)

$2(1.5)$

$134(98.5)$

$$
\begin{gathered}
0.37(0.12-1.16) \\
1.00 \\
13.91(1.38-140.07)
\end{gathered}
$$

$0.69(0.04-4.33)$

1.00

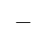

$0.55(0.15-2.02)$

1.00

3.73 (1.26-11.10)

1.00

$0.44(0.10-1.40)$

1.00

$3.03(0.98-9.34)$

1.00

$1.42(0.14-14.22)$

1.00

$0.33(0.04-2.64)$

1.00

$1.82(0.52-6.32)$

1.00

$2.11(0.87-5.14)$

1.00
$25.52(2.27-239.50)$

1.00

$0.95(0.26-3.51)$

$5.75(0.22-148.62)$

1.00

$4.60(0.96-21.97)$

1.00

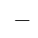

$-$

$13.70(2.36-79.71)$

1.00

$4.04(1.32-12.40)$

1.00

$1.10(0.12-9.84)$

1.00

$2.81(0.25-32.25)$

1.00

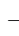

$-$
$100.50(20.10-502.40)$

1.00

${ }^{\text {a }}$ Congential brain anomaly other than microencephaly and hydrocephaly.

NK, not known. 
Table 3

Multivariate analysis of infantile spasms (IS) cases versus normal controls after model fitting and adjusting for variables in each other

\begin{tabular}{lcl}
\hline Variable & OR & $95 \%$ CI \\
\hline Developmental delay before IS & $30.12^{*}$ & $5.58-162.49$ \\
Asphyxia & $29.57^{*}$ & $3.04-287.41$ \\
Congenital cerebral anomaly** & $4.51^{*}$ & $1.02-19.94$ \\
Prematurity & 7.45 & $0.63-88.79$ \\
Febrile seizure & 1.03 & $0.17-6.03$ \\
Age & 1.06 & $0.97-1.17$ \\
Sex & 1.06 & $0.47-2.40$ \\
\hline
\end{tabular}

* Statistically significant, $P<0.05$.

** Cerebral anomaly other than microcephaly and hydrocephaly.

term, and developmental delay (before onset of disease) were at increased risk for IS in Taiwan.

Children with underlying brain disease were highly associated with IS (Ohtahara, 1984; Cusmai et al., 1993; Bobo et al., 1994; Chiron et al., 1996; Rantala et al., 1996; Dulac et al., 1997; Ozawa et al., 1998). Developmental delay before onset of IS may be a sign of preexisting brain injury. These brain insults could result in clinical manifestation of developmental delay and IS. The prognosis of our IS cases revealed that $60 \%$ have suffered from CP or motor handicap and $77 \%$ have developmental delay which may further support this finding.

Few studies have been conducted to elucidate the association between relevant risk factors and IS using comparison group. Only one study, to our knowledge, was conducted to identify risk factors on the basis of case-control studies (Rantala et al., 1996). To test whether subjects that had a family history of seizure were more susceptible to IS than

\section{Table 4}

Multivariate analysis of infantile spasms cases versus disease controls after model fitting and adjusting for variables in each other

\begin{tabular}{lcl}
\hline Variables & OR & $95 \% \mathrm{CI}$ \\
\hline Postterm & $15.61^{*}$ & $1.37-178.26$ \\
Tuberous sclerosis & $9.05^{*}$ & $2.24-36.59$ \\
Age & 0.89 & $0.78-1.01$ \\
Sex & 1.90 & $0.67-5.36$ \\
\hline
\end{tabular}

* Statistically significant, $P<0.05$. those without corresponding family history, Rantala et al. (1996), using CNS infection as the comparison group, demonstrated a 3-fold risk for IS with cryptogenic type. Compared with other epilepsy and febrile seizure, IS was more likely to have $\mathrm{CP}$, microcephaly, hydrocephaly, CNS malformations, perinatal asphyxia, and neonatal seizure. Our results revealed a close relationship between cerebral insults and IS, it suggests that the risk factors of IS may relate to underlying neurologic abnormalities without ethnic difference. In our study, postterm was found to be one of the significant risk factors of IS (OR $=15.61,95 \%$ $\mathrm{CI}=1.37-178.26)$, taking the Disease Control group as a baseline group. However, this was not observed in comparison with the Normal Control group. This may be explained by a recall bias (see below). Otherwise, the importance of postterm in IS requires further investigation.

As displayed in previous studies, whether febrile seizures were associated with IS was still open to debate (Dulac et al., 1993). For example, a family history of epilepsy or febrile seizures is found in $7-17 \%$ of patients with IS (Lacy and Penry, 1976). Febrile seizures were not a significant factor for IS, regardless of using Disease Control group and Normal Control group as baseline groups in this study. The explanation is that febrile seizures can occur as early as 3 months of age and, more often than not, between 6 months and 6 years of age. Peak age for febrile seizures is 18-24 months which is above the age at which IS tends to develop febrile seizures. A febrile seizure in the first few month of life followed by the evolution of IS would actually make one think of severe myoclonic epilepsy (SME) in infancy. However, the clinical presentation of SME lacks typical flexion or extension tonic spasms and EEG revealing spike-and-wave complexes but not hypsarrhythmic which enables one to distinguish SME from IS.

It is unjustified to make the comparison between the IS group and the Disease group for risk factors such as asphyxia and pre-maturity because the disease group may be also more likely to yield asphyxia and pre-maturity than the IS group. This may lead to a false negative association due to such a selection of the disease group as the control group. For example, the OR for asphyxia 
and pre-maturity are estimated as 0.37 and 0.44 are good examples of these cases. The interpretation of results between asphyxia and pre-maturity should be taken with great caution.

From a methodological viewpoint, our study design surpassed previous studies in several aspects. First, a hospital-based case-control study in our study provided an opportunity to identify risk factors that cannot be found using data on case series due to a lack of comparison. Another advantage was the use of two control groups, which enabled us to assess recall bias for some variables that have been commonly observed in retrospective studies since those who had the disease are more able to recall relevant events than those who had not. If one can get consistent results from the two series of controls, the result will be more accurate and convincing. For example, the association of febrile seizures with IS is of borderline statistical significance for both Normal Control and Disease Control group. This suggests that recall bias may be a minor problem. As shown in Table 2, the identical distribution of family history of psychiatric disease, seizure, or epilepsy for the two groups may partially clear the doubt of this problem. In addition, the use of Disease Control group provides an opportunity to investigate the effect of TS or other brain insults on IS. The Disease Control group, using CNS infection as the comparison group, was also used by Rantala et al. (1996).

Our results revealed that the peak age of disease onset is between 3 and 8 months after birth, which was consistent with previous reports in IS (Dulac et al., 1997). These estimates are very helpful for understanding the condition and natural history of IS in Taiwan. Information on age onset plus risk factors can provide an empirical evidence on consultation with parents who are under increased risk of IS. Treatment of IS will be started as soon as possible once identified since it has been regarded as a medical emergency for obtaining best treatment results and long term outcomes (Singer et al., 1980; Fois et al., 1984).

There are two major caveats in our study. Since 25 IS cases were derived from hospital, it could be argued that the risk factors associated with IS cannot be applied to a general population. How- ever, this should not be regarded as a serious issue. The reasonable finding on peak age at onset of IS in our series suggests that it is unlikely that the 25 IS cases are a biased sample. Arguably, 25 cases may lead to a lack of statistical power to test the association between certain risk factor and IS. However, since our results are consistent with the previous findings, the problem of lacking statistical power may not be substantial. In addition, Fisher exact test (statistical method for small sample) has been applied to assess the significance of the results for variables with expected number less than five in some cells that are still significant (data not shown). However, the estimated OR accompanied with a wide confidence may still indicate the limitation of small sample sizes in this study. From the statistical testing viewpoint, small sample sizes may render the results become conservative and insignificant. Namely, no significant results may be due to a lack of power and should be clarified whereas a significant association must become more salient if greater sample sizes are used. We believe our results are conservative.

In conclusion, we elucidate underlying neurological abnormalities as the putative risk factors associated with IS. Our results not only make a clear understanding of disease burden of IS in this society but clarify more etiology of IS than before.

\section{Acknowledgements}

This work was supported by NSC 89-2314-B002-145 form National Science Council, Taiwan.

\section{References}

Bobo, J.K., Thapa, P.B., Anderson, J.R., Gale, J.L., 1994. Acute encephalopathy and seizures rates in children under age two years in Oregon and Washington State. Am. J. Epidemiol. 149, 27-38.

Chiron, C., Nabbout, R., Pinton, F., Nuttin, C., Dulac, O., Syrota, A., 1996. Brain functional imaging SPECT in agyria-pachygyria. Epilepsy Res. 24, 109-117.

Cowan, L.D., Bodensteiner, J.B., Leviton, A., Doherty, L., 1989. Prevalence of the epilepsies in children and adolescents. Epilepsia 30, 94-106. 
Commission on Classification and Terminology of the International League Against Epilepsy, 1989. Proposal for Revised Classification on Epilepsies and Epileptic Syndromes. Epilepsia 30, 389-3919849.

Cusmai, R., Ricci, S., Pinard, J.M., Plouin, P., Fariello, G., Dulac, O., 1993. West syndrome due to perinatal insults. Epilepsia 34, 738-742.

Debrock, C., Preux, P.M., Houinato, D., Druet-Cabanac, M., Kassa, F., Adjien, C., Avode, G., Denis, F., Boutros-Toni, F., Dumas, M., 2000. Estimation of the prevalence of epilepsy in the Benin region of Zinvie using the capture-recapture method. Int. J. Epidemiol. 29, 330-335.

Dulac, O., Feingold, J., Plouin, P., Chiron, C., Pajot, N., Ponsot, G., 1993. Genetic predisposition to West syndrome. Epilepsia 34, 732-737.

Dulac, O., Plouin, P., Schlumberger, E., 1997. Infantile spasms. In: Wyllie, E. (Ed.), The Treatment of Epilepsy: Principle and Practice, 2nd Edition. William \& Wilkins, Baltimore, MD, pp. 540-572.

Filipek, P.A., Krishnamoorthy, K.S., Davis, K.R., Kuehnle, K., 1987. Focal cerebral infarction in the newborn: a distinct entity. Pediatr. Neurol. 3, 141-147.

Fois, A., Malandrini, F., Balestri, P., Giorgi, D., 1984. Infantile spasms-long term results of ACTH treatment. Eur. J. Pediatr. 142, 51-55.

Gibbs, F.A., Gibbs, E.L., 1952. Atlas of Electroencephalography, II. Epilepsy. Addision-Wesley, Reading, MA.

Hanjal, B.L., Sahebkar-Moghaddam, F., Barnwell, A.J., Barkovich, A.J., Ferriero, D.M., 1999. Early prediction of neurologic outcome after perinatal depression. Pediatr. Neurol. 21, 788-793.

Hauser, W.A., 1994. The prevalence and incidence of convulsion disorders in children. Epilepsia 35 (Suppl. 2), S1-S6.

Hou, J.W., Wang, P.J., Wang, T.R., 1994. Tuberous sclerosis in children. Acta Paediatr. Sin. 35, 102-107.

Howitz, P., Platz, P., 1978. Infantile spasms and HLA antigens. Arch. Dis. Child. 53, 680-682.

Hrachovy, R.A., Frost, J.D., Kellaway, P., 1984. Hypsarrhythmia: variations on the theme. Epilepsia 25, 317325.
Hrachovy, R.A., Frost, J.D. Jr, 1989. Infantile spasms: a disorder of the developing nervous system. In: Kellaway, P., Noebels, J.L. (Eds.), Problems and Concepts in Developmental Neurophysiology. Johns Hopkins University Press, Baltimore, MD, pp. 131-147.

Kellaway, P., Hrachovy, R.A., Frost, J.D., Zion, T., 1979. Precise characterization and quantification of infantile spasms. Ann. Neurol. 6, 214-218.

Lacy, J.R., Penry, J.K., 1976. Infantile Spasms. Raven Press, New York.

Luthvigsson, P., Olafsson, E., Sigurthardottir, S., Hauser, W.A., 1994. Epidemiologic features of infantile spasms in Iceland. Epilepsia 35, 802-805.

Ohtahara, S., 1984. Seizure disorder in infancy and childhood. Brain Dev. 6, 509-519.

Ozawa, H., Hashimoto, T., Endo, T., Kato, T., Furusho, J., Suzuki, Y., Takada, E., Ogawa, Y., Takashima, S., 1998. West syndrome with periventricular leukomalacia: a morphometric MRI study. Pediatr. Neurol. 19, 358363.

Riikonen, R., Donner, M., 1979. Incidence and etiology of infantile spasms from 1960 to 1976. Dev. Med. Child. Neurol. 21, 333-343.

Rantala, H., Shields, W.D., Christenson, P.D., Nielsen, C., Buch, D., Jacobsen, V., Zachau-Christiansen, B., Uhari, M., Cherry, J.D., 1996. Risk factors of Infantile spasms compared with other seizures in children under 2 years of age. Epilepsia 37, 362-366.

Shevell, M.I., Majnemer, A., Miller, S.P., 1999. Neonatal neurologic prognostication: the asphyxia term newborn. Pediatr. Neurol. 21, 776-784.

Singer, W.D., Rabe, E.F., Haller, J.S., 1980. The effect of ACTH therapy upon infantile spasms. J. Pediatr. 96, 485489.

Trevathan, E., Murphy, C.C., Allsopp, M.Y., 1999. The descriptive epidemiology of infantile spasms among Atlanta children. Epilepsia 40, 748-751.

Watanabe, K., 1998. West Syndrome: etiological and prognostic aspects. Brain Dev. 20, 1-8. 